Edinburgh 2000/19

hep-lat/0009008

September 2000

\title{
THE TOPOLOGICAL SUSCEPTIBILITY AND PION DECAY CONSTANT FROM LATTICE QCD
}

\author{
UKQCD Collaboration: A. HART ${ }^{a}$ \\ Dept. of Physics and Astronomy, Univ. of Edinburgh, Edinburgh, Scotland \\ and M. TEPER \\ Theoretical Physics, Univ. of Oxford, 1 Keble Road, Oxford, England
}

\begin{abstract}
We study the topological susceptibility, $\chi$, in two flavour lattice QCD.11 We find clear evidence for the expected suppression of $\chi$ at small quark mass. The estimate of the pion decay constant, $f_{\pi}=105 \pm 5_{-10}^{+18} \mathrm{MeV}$, is consistent with the experimental value of approximately $93 \mathrm{MeV}$. We compare $\chi$ to the large- $N_{c}$ prediction and find consistency over a large range of quark masses.
\end{abstract}

The ability to access the non-perturbative sectors, and to vary parameters fixed in Nature has made lattice Monte Carlo simulation a valuable tool for investigating the rôle of topological excitations in QCD and related theories.

The topological susceptibility is the squared expectation value of the topological charge, normalised by the volume

$$
\chi=\frac{\left\langle Q^{2}\right\rangle}{V}, \quad Q=\frac{1}{32 \pi^{2}} \int d^{4} x \frac{1}{2} \varepsilon_{\mu \nu \sigma \tau} F_{\mu \nu}^{a}(x) F_{\sigma \tau}^{a}(x) .
$$

Sea quarks induce an instanton-anti-instanton attraction which in the chiral limit becomes stronger, suppressing $Q$ and $\chi^{3}$

$$
\chi=\Sigma\left(m_{u}^{-1}+m_{d}^{-1}\right)^{-1}, \quad \text { where } \quad \Sigma=-\lim _{m_{q} \rightarrow 0} \lim _{V \rightarrow \infty}\langle 0|\bar{\psi} \psi| 0\rangle
$$

is the chiral condensate. 1 We assume $\langle 0|\bar{\psi} \psi| 0\rangle=\langle 0|\bar{u} u| 0\rangle=\langle 0|\bar{d} d| 0\rangle$ and neglect contributions of heavier quarks. The Gell-Mann-Oakes-Renner relation,

$$
f_{\pi}^{2} m_{\pi}^{2}=\left(m_{u}+m_{d}\right) \Sigma+\mathcal{O}\left(m_{q}^{2}\right) \quad \Rightarrow \quad \chi=\frac{f_{\pi}^{2} m_{\pi}^{2}}{2 N_{f}}+\mathcal{O}\left(m_{\pi}^{4}\right)
$$

for $N_{f}$ light flavours, in a convention where the experimental value of the pion decay constant $f_{\pi} \simeq 93 \mathrm{MeV}$. Eq. 3 holds in the limit $f_{\pi}^{2} m_{\pi}^{2} V \gg 1$, which is satisfied by all our lattices. The higher order terms ensure that $\chi \rightarrow \chi^{\mathrm{qu}}$, the quenched value, as $m_{q}, m_{\pi} \rightarrow \infty$. We find, however, that our measured values are not very much smaller than $\chi^{\mathrm{qu}}$, so we must consider two possibilities.

$\overline{{ }^{a}}$ Talk presented at the Confinement IV meeting, Vienna, July 2000. 
Figure 1: The measured topological susceptibility, with interpolated quenched points at the same $\hat{r}_{0}$ and fits independent of the quenched points.
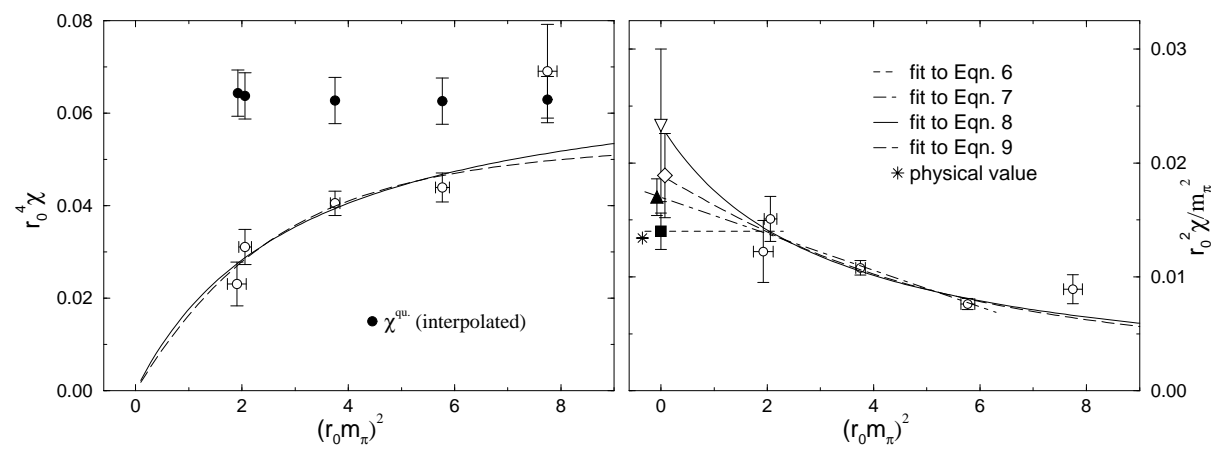

Firstly, there are phenomenological reasons 0.6 for believing that QCD is 'close' to $N_{c}=\infty$ and in the case of gluodynamics even $\mathrm{SU}(2)$ is demonstrably close to $\mathrm{SU}(\infty)$. 2 . 2 Fermion effects are non-leading in $N_{c}$, so we expect $\chi \rightarrow \chi^{\text {qu }}$ for any fixed value of $m_{q}$ as the number of colours $N_{c} \rightarrow \infty$. For small $m_{q}$ we expect Eq. 4 to hold, with $\chi^{\infty}, f_{\infty}$ the quantities at leading order in $N_{c}$. 4 Alternatively, our $m_{q} \simeq m_{\text {strange }}$ and perhaps higher order terms are important. In the absence of a QCD prediction, Eq. 5 interpolates between Eq. 3 and the quenched limit.

$$
\chi=\frac{\chi^{\infty} m_{\pi}^{2}}{\frac{2 N_{f} \chi^{\infty}}{f_{\infty}^{2}}+m_{\pi}^{2}}(4), \quad \chi=\frac{f_{\pi}^{2}}{\pi N_{f}} m_{\pi}^{2} \arctan \left(\frac{\pi N_{f}}{f_{\pi}^{2}} \chi^{\mathrm{qu}} \frac{1}{m_{\pi}^{2}}\right)
$$

Measurements of $\chi$ were made on a number of ensembles of $N_{f}=2$ lattice field configurations produced by the UKQCD Collaboration An SU(3) Wilson gauge action is coupled to clover improved Wilson fermions. 6 The UKQCD ensembles have two notable features. The improvement is fully non-perturbative, with discretisation errors being quadratic rather than linear in the lattice spacing. Second, the couplings are chosen to maintain an approximately constant lattice spacing (as defined by the Sommer scale, $r_{0}=0.49 \mathrm{fm}$ ) as the quark mass is varied. This is important, as the susceptibility in gluodynamics varies with the lattice spacing as $\hat{r}_{0}^{4} \hat{\chi}=0.072-0.208 / \hat{r}_{0}^{2}$ in competition with the variation with $m_{q}$. The topological susceptibility is measured from the gauge fields after cooling to remove the UV noise. We plot these data in Fig. 1 along with the interpolated $\chi^{\mathrm{qu}}$ at an equivalent lattice spacing from the above formula for comparison, which vary little owing to the the UKQCD matching. The behaviour with $M \equiv\left(\hat{r_{0}} \hat{m}_{\pi}\right)^{2}$ is qualitatively as expected and, more quantita- 
Table 1: $\quad$ Fits to the $N_{\text {fit }}$ most chiral points of $\hat{\chi}$.

\begin{tabular}{|c|c|c|c|l|c|}
\hline Fit & $N_{\text {fit }}$ & $c_{0}$ & $c_{1}$ & $\chi^{2} /$ d.o.f. & $\hat{r}_{0} \hat{f}_{\pi}$ \\
\hline Eq. 6 & 2 & $0.0140(16)$ & - & 0.805 & $0.237(14)$ \\
Eq. 6 & 3 & $0.0112(6)$ & - & 2.202 & $0.212(6)$ \\
Eq. 6 & 4 & $0.0091(4)$ & - & 9.008 & - \\
\hline Eq. 7 & 3 & $0.0176(35)(4)$ & $-0.0018(10)(1)$ & 0.964 & $0.265(27)$ \\
Eq. 7 & 4 & $0.0170(16)(1)$ & $-0.0016(4)(0)$ & 0.502 & $0.261(13)$ \\
Eq. 7 & 5 & $0.0147(14)(1)$ & $-0.0011(3)(0)$ & 2.965 & $0.242(12)$ \\
\hline \hline Fit & $N_{\text {fit }}$ & $c_{0}$ & $c_{3}$ & $\chi^{2} /$ d.o.f. & $\hat{r}_{0} \hat{f}_{\pi}$ \\
\hline Eq. 8 & 3 & $0.0208(87)(12)$ & $0.0844(427)(35)$ & 1.013 & $0.288(61)$ \\
Eq. 8 & 4 & $0.0272(85)(18)$ & $0.0632(114)(6)$ & 0.895 & $0.329(53)$ \\
Eq. 8 & 5 & $0.0233(66)(10)$ & $0.0717(147)(3)$ & 1.847 & $0.305(44)$ \\
\hline Eq. 9 & 3 & $0.0186(53)(7)$ & $0.0576(175)(6)$ & 0.990 & $0.273(40)$ \\
Eq. 9 & 4 & $0.0209(42)(7)$ & $0.0506(55)(5)$ & 0.682 & $0.289(30)$ \\
Eq. 9 & 5 & $0.0189(36)(5)$ & $0.0550(69)(6)$ & 1.929 & $0.275(27)$ \\
\hline
\end{tabular}

tively, we attempt fits motivated by Eqs. 3, 4, 5:

$$
\frac{{\hat{r_{0}}}^{2} \hat{\chi}}{M}=c_{0} \quad(6), c_{0}+c_{1}\left(\hat{r_{0}} \hat{m}_{\pi}\right)^{2} \quad(7), \frac{c_{0} c_{3}}{c_{3}+c_{0} M} \quad(8), \frac{2 c_{0}}{\pi} \tan ^{-1}\left(\frac{\pi c_{3}}{2 c_{0} M}\right)
$$

We include progressively less chiral points until the fit becomes unacceptably bad in Table 1 . We note the wide range fitted simply by including an $m_{\pi}^{4}$ term, and the consistency of our data with large- $N_{c}$ predictions. The stability and similarity of the fits motivates us to use $c_{0}$ from Eq. 7 to estimate $f_{\pi}=105 \pm$ $5_{-10}^{+18} \mathrm{MeV}$, with variation between other fits providing the second, systematic error, and in good agreement with the experimental value $\simeq 93 \mathrm{MeV}$.

\section{References}

1. See also A. Hart, M. Teper, Nucl. Phys. B (Proc. Suppl.) 83-84 (2000) 476 hep-lat/9909072]; hep-ph/0004180; UKQCD Collab., in prep.

2. For a recent review, see M. Teper, Nucl. Phys. B (Proc. Suppl.) 83-84 (2000) 146 hep-lat/9909124.

3. P. Di Vecchia, G. Veneziano, Nucl. Phys. B 171 (1980) 253.

4. H. Leutwyler, A. Smilga, Phys. Rev. D 46 (1992) 5607.

5. G. 't Hooft, Nucl. Phys. B72 (1974) 461.

6. E. Witten, Nucl. Phys. B160 (1979) 57.

7. M. Teper, Phys. Rev. D 59 (1999) 014512 hep-lat/9804008; hepth/9812187.

8. J. Garden (UKQCD), Nucl. Phys. B (Proc. Suppl.) 83 (2000) 165 hep-lat/9909066.

9. R. Sommer, Nucl. Phys. B411 (1994) 839 hep-lat/9310022. 